\title{
PARTICLE COLLISIONS IN ERGOREGION OF BRANEWORLD KERR BLACK HOLE
}

\author{
Saeed Ullah Khan ${ }^{1 *}$ Misbah Shahzadi ${ }^{2 \dagger}$ and Jingli $\operatorname{Ren}^{1 \neq}$ \\ ${ }^{1}$ School of Mathematics and Statistics, Zhengzhou University, Zhengzhou 450001, China. \\ ${ }^{2}$ Department of Mathematics, COMSATS University Islamabad, Lahore Campus-54000, Pakistan.
}

\begin{abstract}
This paper explores the neutral particle motion and collisional Penrose process in ergoregion of the braneworld Kerr black hole. We analyze the properties of event horizon, ergosphere and static limit. The particle collision in ergoregion via the Penrose process is investigated. Furthermore, we study the negative energy states and show that the sign of particle energy can be uniquely determined by the sign of angular momentum. In addition, we study the Wald inequality to determine the limits of energy extraction via the Penrose process and also find lower bound of the irreducible mass. The expression for the efficiency of energy extraction from the brane Kerr black hole is found. Finally, we compare our results with that obtained from the Kerr black hole. It is concluded that efficiency increases with the increase of rotation as well as brane parameter $b$ of the black hole.
\end{abstract}

Keywords Black hole Physics · Gravitation · Collisions

\section{Contents}

1 Introduction 2

2 Braneworld Kerr Black Hole

2.1 Ergoregion, Horizon and Static Limit . . . . . . . . . . . . . . . . . . . 3

3 Particle Dynamics

3.1 Angular Velocity of a Particle in Ergoshpere . . . . . . . . . . . . . . . . . . . . 7

4 Collisional Process in Ergoregion $\quad 8$

4.1 Negative Energy States . . . . . . . . . . . . . . . . . . . . . . 8

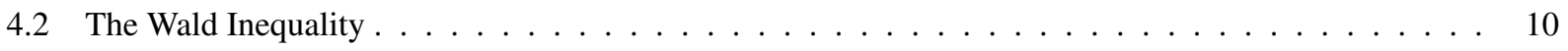

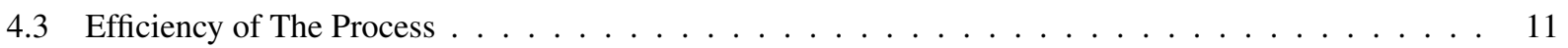

5 Concluding Remarks

*saeedkhan.u@gmail.com

${ }^{\dagger}$ misbahshahzadi51@gmail.com

†renjl@zzu.edu.cn 


\section{Introduction}

In recent years, researchers have made great contributions to the higher-dimensional string as well as M-theories, which are among the most aspiring approaches to the higher dimensional gravity theories [1, 2]. These theories describe gravity as a truly higher dimensional interaction that becomes effectively 4D at low enough energies. The braneworld models have been inspired by these theories, where the observable Universe is a 3-brane, on which the standard-model (non-gravitational) matter fields are confined, while the gravity fields enter into the extra spatial dimensions. The extra dimensions could modify the properties of a black hole $(\mathrm{BH})$ and may have much larger size than that of the Planck length scale $\left(l_{p} \sim 10^{-33} \mathrm{~cm}\right)$ [3]. It is possible that these extra dimensions could have infinite size, just like in case of the braneworld model of Randall and Sundrum comprise of one extra spatial dimension [4]. Consequently, these models may provide an effective solution to the hierarchy problems of the electroweak and quantum gravity scales, as these scales could become to be of the same orders $(\sim \mathrm{TeV})$ due to the large scale extra dimensions. Hence, the braneworld models can be tested by future collider experiments quite well, inclusive of the hypothetical mini BH construction of the TeV- energy scales [5, 6]. In addition, the angular momentum of a BH in the vicinity of 4D general relativity (GR) is limited by the Kerr bound but in string theory, the required bound could split and compact objects like BH can spin faster [7].

In the last few decades, a number of scientific research has been carried out to study the dynamics around ReissnerNordström (RN), Kerr-Newman (KN) and modified BHs [8, 9, 10, 11, 12], which one can directly apply to the braneworld models, considering the effects of only positive tidal charge. Dadhich et al. [13] deduced that the RN spacetime is the exact solution of effective Einstein equations on brane, re- defined as a BH with the effects of tidal charge rather than the electric charge. Kotrlová et al. [14] by examining the braneworld model, inferred that mass of the neutron star reduces due to the existence of negative tidal charge. Pugliese et al. [15, 16] studied the RN spacetime and extrapolates the existence of circular orbit with vanishing angular momentum. Exploring the circular geodesics in Kerr-Newman BH, it is concluded that BHs and naked singularities can be altered using the structure of stability's regions [17]. The braneworld models were also studied by many researchers considering both negative as well as positive tidal charge effects [18, 19, 20, 21]. Stuchlík and Hledík [22] studied the properties of RN BH and naked singularity with non-zero cosmological constant and determined the photon escape cones. Grib and Pavlov [23] explored the energy bounds within ergosphere of a $\mathrm{BH}$ and found that there is no extreme $\mathrm{BH}$ having a critical value of intrinsic angular momentum of the BH rotation. Nakao et al. [24] concluded that the Kerr superspinars can be stable against linear perturbations whereas, BHs on the Randall- Sundrum brane could be stable against each type of perturbations [25]. Stuchlik and Kolos [26] studied the chaotic scattering around a BH immersed in uniform magnetic field and observed that the strong acceleration of ionized particles towards ultra-relativistic velocities could be preferred in the direction nearby the magnetic field lines.

It is widely known that similar to the astrophysical conditions, the BH electric charge becomes negligible or vanished on small intervals of time due to its neutralization of accreting preferentially oppositely charged particles from an ionized matter of the accretion disc [27, 28]. This statement is also true in case of the braneworld models, that's why it is sufficient to examine the properties of brane Kerr BHs enriched with its tidal charge effects only. The consequences of tidal charge effects were also studied by some researchers in recent years for optical lensing in the weak field limits [29] and in the time delay effects [30]. It is observed that for the brane Kerr BH with fixed rotational parameter, the increasing values of negative tidal charge strengthens the relativistic effects [31]. Schee and Stuchlík [32] deduced that the profiled lines in the framework of braneworld Kerr spacetime depend on spin as well as brane parameter and become wider by lowering the negative tidal charge.

The process of energy extraction from rotating BHs is among the significant and aspiring problems in the fields of GR as well as in astrophysics. Penrose [33] introduce a truly accurate mechanism to extract energy from a rotating $\mathrm{BH}$ and related to the existence of the negative energy in ergoregion. It is found that energy extraction could be greater in case of the higher dimensional BHs as compared to the energy extortion from 4D Kerr BH [34]. It is deduced that more energy can be extracted with rotating particle as compared to the non-rotating case [35].

The Efficiency of energy extraction from a rotating BH via the Penrose process could be explained as (gain in energy)/(input energy). Efficiency of the Penrose process gets minimized around KN BH due to the vicinity of charge in comparison with the maximum efficiency limit of $20.7 \%$ for that of the Kerr BH [36]. Parthasarathy et al. [37] by studying rotating $\mathrm{BH}$ under the effect of magnetic field, extrapolate that, efficiency of the Penrose process could reach up to $100 \%$, if an incoming particle splits near the static limit. Liu et al. [38] found that in the case of non-Kerr BH deformation parameter increases the efficiency of energy extraction. Investigation of the Penrose mechanism around a regular rotating $\mathrm{BH}$ shows decreasing behavior in the efficiency of the energy extraction for increasing values of the electric charge [39]. Liu and Liu [40] studied the Penrose mechanism with rotating particles and deduced that efficiency of the energy extraction monotonically increases as the particle rotation increases. Dadhich et al. [41] explored the Penrose process in the presence of magnetic field and concluded that efficiency of the process increases with the 
magnetic charge. Shahzadi et al. [42] explored the particle motion near Kerr-MOG (modified theory of gravity) BH and found that the efficiency of energy extraction can be enhanced with the increase of the dimensionless parameter of the theory.

In this article, we investigate the neutral particle motion and the collisional Penrose process within ergoregion of the braneworld Kerr BH. The article is organized as follows: the coming section will briefly summarize the braneworld Kerr $\mathrm{BH}$, its properties and effects of the tidal charge on ergoregion, static limit and on horizons of the $\mathrm{BH}$. In section 3 . we derive the equations of motion and discuss the angular velocity of particles in the vicinity of ergoregion. In the next section, we study the collisional Penrose process and investigate the negative energy states, Wald inequality as well as the efficiency of energy extraction. Finally, we summarize our results in the last section.

\section{Braneworld Kerr Black Hole}

The braneworld kerr BH is an axially symmetric, stationary and asymptotically flat solution of the effective Einstein equations on the brane. The spacetime geometry of the braneworld Kerr BH could be outlined by the metric with Boyer-Lindquist coordinates as [43]

$$
d s^{2}=g_{t t} d t^{2}+2 g_{t \phi} d t d \phi+g_{r r} d r^{2}+g_{\theta \theta} d \theta^{2}+g_{\phi \phi} d \phi^{2}
$$

with

$$
\begin{aligned}
g_{t t} & =-\left(\frac{\Delta-a^{2} \sin ^{2} \theta}{\Sigma}\right), \quad g_{r r}=\frac{\Sigma}{\Delta}, \quad g_{\theta \theta}=\Sigma, \\
g_{\phi \phi} & =\frac{\sin ^{2} \theta}{\Sigma}\left[\left(r^{2}+a^{2}\right)^{2}-\Delta a^{2} \sin ^{2} \theta\right], \\
g_{t \phi} & =\frac{a \sin ^{2} \theta}{\Sigma}\left[\Delta-\left(r^{2}+a^{2}\right)\right],
\end{aligned}
$$

where

$$
\Delta=r^{2}-2 M r+a^{2}+b, \quad \Sigma=r^{2}+a^{2} \cos ^{2} \theta
$$

Here, $M$ and $a$ represents the mass and spin parameter of the $\mathrm{BH}$, respectively. In the case of braneworld Kerr BH, the impact of tidal effects from the bulk is expressed by a single parameter known as the tidal charge $b$, which can take both positive as well as negative values. It can be observed that metric (1) exactly looks like the KN BH with the replacement of $b$ by $Q^{2}$ (representing electric charge of the KN BH). Moreover, it is worthwhile to note that for different values of the parameter $b$, Eq. (1) assumes special cases, i.e., it reduced to the Kerr BH if $b=0$; for $b>0$, we obtained the KN $\mathrm{BH}$; and finally for $b<0$, it reduced into the non-standard KN BH with negative tidal effects. Furthermore, for both $a$, $b=0$, the metric (1) reduced to the Schwarzschild $\mathrm{BH}$.

\subsection{Ergoregion, Horizon and Static Limit}

The ergoregion (section lies between the horizons and the static limit of a BH) play an important role in astrophysics, as the Hawking radiation could be examined in this region. Moreover, the ergoregion of a BH is also momentous because of the Penrose process, as it takes place in this region. The static limit surface $\left(r_{e s}\right)$ is also termed as the infinite redshift surface, in which the time-translation killing vector becomes null. When the time-like geodesics crossed the static limit surface they changed into the space-like geodesics. On substituting $g_{t t}=0$, the ergosphere turns out to be

$$
r_{e s}=M \pm \sqrt{M^{2}-a^{2} \cos ^{2} \theta-b}
$$

The existence of static limit requires that

$$
M^{2} \geq a^{2} \cos ^{2} \theta+b .
$$

The horizons of Eq. (1) can be found by solving $\Delta=0$ as

$$
r_{ \pm}=M \pm \sqrt{M^{2}-a^{2}-b}
$$

The event horizon will exist if

$$
M^{2} \geq a^{2}+b
$$

The effects of positive tidal charge tend to reduce gravitational field and in this case, similar horizons structure can be found as in the $\mathrm{KN} \mathrm{BH}$. However, the negative tidal charge may lead us to some new interesting features of the BH. For $a \rightarrow M$ and $b<0$, the horizon radius $r_{+} \rightarrow(M+\sqrt{-b})>M$, and this condition does not hold in GR. The 

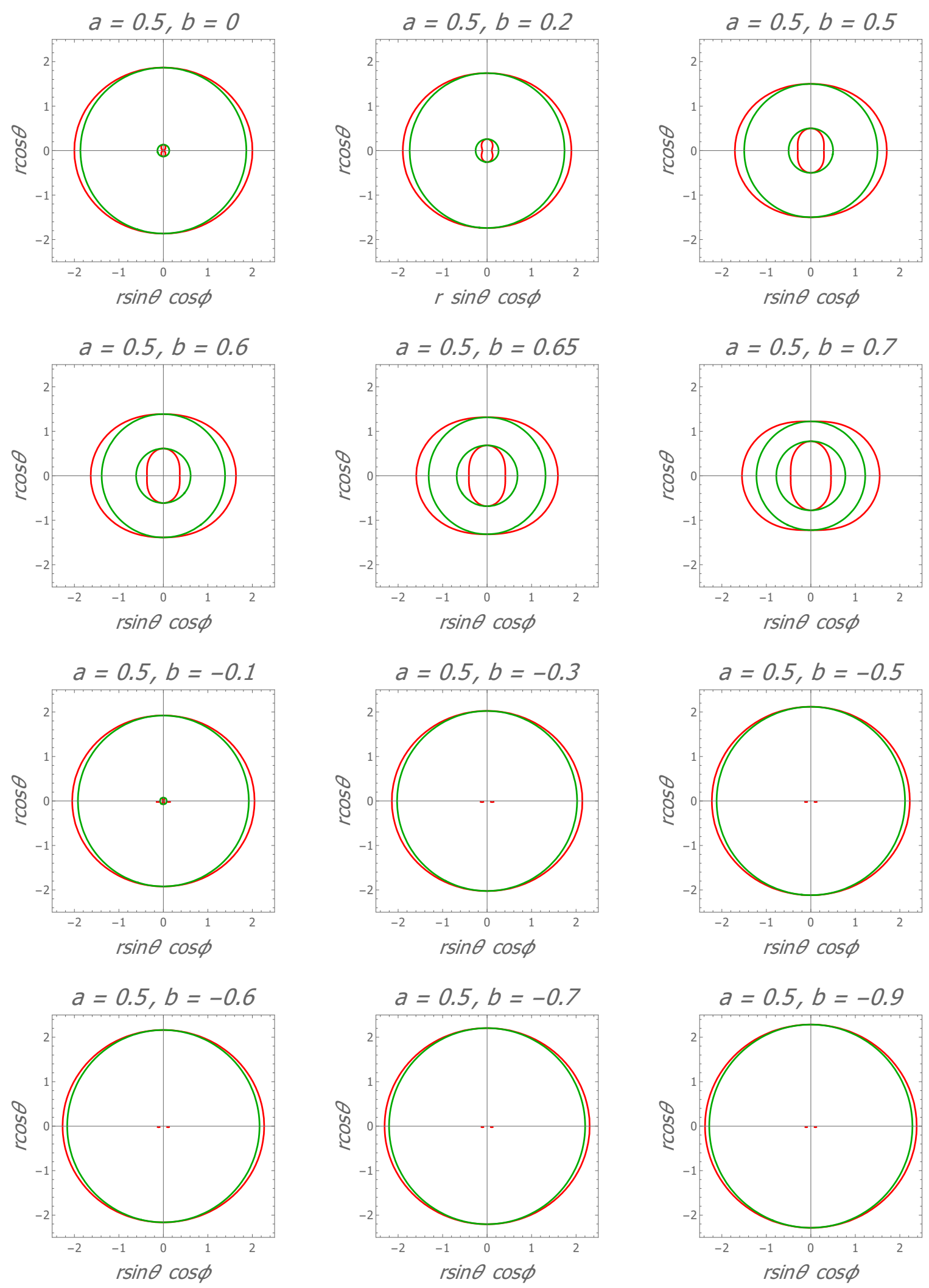

Figure 1: The ergosphere and horizons in the xz-plane for different values of tidal charge $b$. 

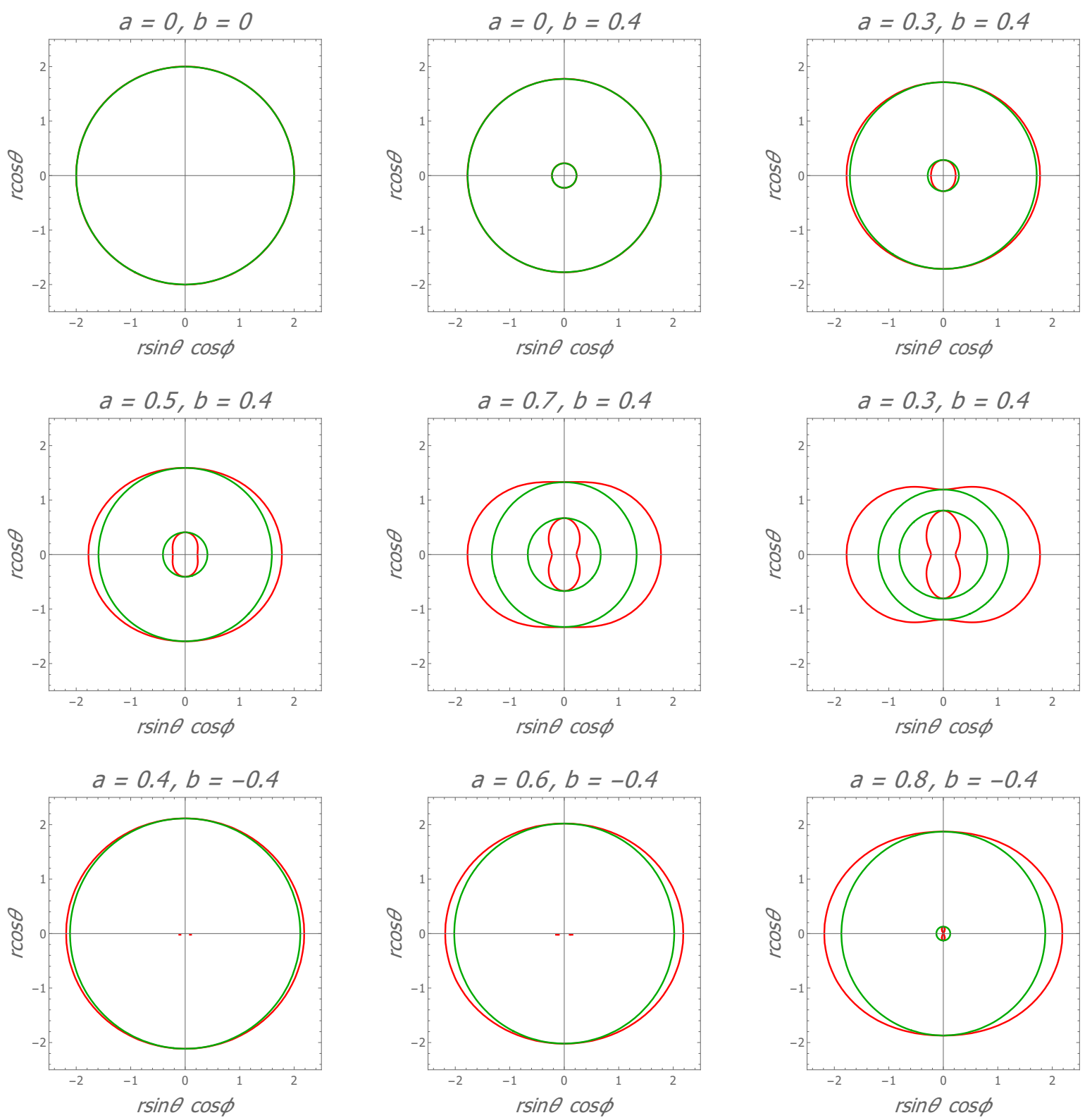

Figure 2: The ergosphere and horizons in the xz-plane for different values of spin parameter $a$. 
Table 1: The ergoregion $\left(\delta=r_{+e s}-r_{+}\right)$, event horizon $\left(r_{+}\right)$and static limit $\left(r_{+e s}\right)$.

\begin{tabular}{|c|c|c|c|c|c|c|c|c|}
\hline & \multicolumn{2}{|r|}{$a=0.2$} & \multicolumn{2}{|r|}{$a=0.3$} & \multicolumn{2}{|r|}{$a=0.4$} & \multicolumn{2}{|r|}{$a=0.5$} \\
\hline$b$ & $r_{+}$ & $r_{+e s} \quad \delta$ & $r_{+}$ & $r_{+e s}$ & $r_{+}$ & $r_{+e s}$ & $r_{+}$ & $r_{+e s}$ \\
\hline-0.7 & 2.2884 & 2.29620 .0078 & 2.2689 & 2.28660 .0177 & 2.2410 & 2.27280 .0318 & 2.204 & 2.25500 .0508 \\
\hline-0.5 & 2.2083 & 2.21660 .0083 & 2.1874 & 2.20620 .0188 & 2.1576 & 2.19170 .0341 & 2.118 & 2.17260 .0550 \\
\hline-0.3 & 2.1225 & 2.13140 .0089 & 2.1000 & 2.12030 .0203 & 2.0677 & 2.10500 .0368 & 2.024 & 2.08400 .0593 \\
\hline-0.1 & 2.0296 & 2.03920 .0097 & 2.0050 & 2.02710 .0221 & 1.9695 & 2.01000 .0404 & 1.922 & 1.98720 .0652 \\
\hline 0.0 & 1.9798 & 1.99000 .0102 & 1.9540 & 1.97720 .0233 & 1.9165 & 1.95920 .0427 & 1.866 & 1.93540 .0694 \\
\hline 0.1 & 1.9274 & 1.93810 .0107 & 1.9000 & 1.92470 .0247 & 1.8602 & 1.90550 .0453 & 1.806 & 1.88030 .0741 \\
\hline 0.3 & 1.8124 & 1.82470 .0123 & 1.7810 & 1.80930 .0283 & 1.7349 & 1.78740 .0526 & 1.670 & 1.75830 .0875 \\
\hline 0.5 & 1.6782 & 1.69280 .0146 & 1.6403 & 1.67450 .0342 & 1.5831 & 1.64810 .0650 & 1.500 & 1.61240 .1124 \\
\hline 0.7 & 1.5100 & 1.52920 .0192 & 1.4583 & 1.50500 .0467 & 1.3742 & 1.46900 .0948 & 1.223 & 1.41830 .1947 \\
\hline
\end{tabular}

extremal condition can be found at $M^{2}=a^{2}+b$ and in case of the negative tidal charge, the extreme horizon $\left(r_{+}=M\right)$ correspond to the BH with $b=-M^{2}$ and $a=\sqrt{2} M$ can be obtained. Therefore, bulk effects on the brane could provide the mechanism for spinning up the $\mathrm{BH}$ on the brane, consequently, its spin parameter exceeds its mass and this type of situation is impossible in GR.

The numerical values of event horizon, static limit and ergoregion of the braneworld Kerr BH at different values of $b$ and $a$, are given in Table. 1, and the corresponding structures are depicted in Figs. 1 and 2. It is observed that the sign of tidal charge has significant effects on the shape of ergoregion as well as on the horizons. Figure 1 shows that for positive values of the tidal charge the ergoregion becomes thick, while both of its radii for the static limit surface and event horizon decreases. On the other hand, for negative values of the tidal charge, the ergoregion decreases whereas the horizons increases. In Fig. 2 it is demonstrated that the area of ergoregion increases by increasing the values of both $a$ and $b$ and its radii get decreases. In addition, it is found that the braneworld Kerr BH has greater radii and thicker ergoregion as compared to the Kerr BH.

\section{Particle Dynamics}

We assume the neutral particle motion in background of the braneworld Kerr BH and limited our investigation to the case of orbits situated on an equatorial plane. The governing equation of geodesics can be acquired using the Lagrangian equation as

$$
\mathscr{L}=\frac{1}{2} g_{\mu \eta} \dot{x}^{\mu} \dot{x}^{\eta},
$$

where dot means $\partial / \partial \tau\left(\tau\right.$ is the proper time) and $\dot{x}^{\mu}$ represents the four-velocity. The generalized momenta for 11 can be written as

$$
\begin{aligned}
-p_{t} & =g_{t t} \dot{t}+g_{t \phi} \dot{\phi}=E, \\
p_{\phi} & =g_{t \phi} \dot{t}+g_{\phi \phi} \dot{\phi}=L, \\
p_{r} & =g_{r r} \dot{r},
\end{aligned}
$$

where $E$ and $L$ are interpreted as the energy and angular momentum of a particle associated with the Killing vector fields $\xi_{t}=\partial_{t}$ and $\xi_{\phi}=\partial_{\phi}$, respectively. Since the Lagrangian is independent of the coordinates $t$ and $\phi$, so $p_{t}$ and $p_{\phi}$ are conserved along geodesics and hence specifies the stationary as well as axisymmetric properties of the Kerr braneworld BH. From Eqs. (5) and (6), we obtain

$$
\begin{aligned}
\dot{t} & =\frac{1}{r^{2} \Delta}\left[E a^{2}(b-r(2 M+r))+a L(b-2 M r)-E r^{4}\right], \\
\dot{\phi} & =\frac{1}{r^{2} \Delta}[E a(b-2 M r)+L(b+r(r-2 M))] .
\end{aligned}
$$

The Hamiltonian for neutral particle motion can be written as

$$
H=p_{t} \dot{t}+p_{r} \dot{r}+p_{\phi} \dot{\phi}-\mathscr{L} .
$$


For Eq. (1), the above equation turns out to be

$$
\begin{aligned}
2 H & =-\left(g_{t t} \dot{t}+g_{t \phi} \dot{\phi}\right) \dot{t}+\left(g_{t \phi} \dot{t}+g_{\phi \phi} \dot{\phi}\right) \dot{\phi}+g_{r r} \dot{r}^{2} \\
& =E \dot{t}+L \dot{\phi}+\frac{r^{2}}{\Delta} \dot{r}^{2}=\varepsilon=\text { constant },
\end{aligned}
$$

where $\varepsilon=-1,0,1$ specify the timelike, null (lightlike) and spacelike geodesics. Substituting Eqs. (7) and (8) into (9), we find the radial equation of motion

$$
\dot{r}^{2}=E^{2}+\frac{1}{r^{4}}(2 M r-b)(a E-L)^{2}+\frac{1}{r^{2}}\left(a^{2} E^{2}-L^{2}\right)+\varepsilon \frac{\Delta}{r^{2}} .
$$

Eqs. (7)-(10) are very important as they can be used to discuss different properties associated with the particle motion near this $\overrightarrow{\mathrm{BH}}$.

\subsection{Angular Velocity of a Particle in Ergoshpere}

In this section, we are interested to investigate particle angular velocity $(\Omega=d \phi / d t)$ and its limitations within the premises of ergosphere with the condition of $d s^{2} \geq 0$. Hence

$$
g_{t t} d t^{2}+g_{t \phi} d t d \phi+g_{\phi \phi} d \phi^{2} \geq 0
$$

and the angular velocity must satisfy the constraints $\Omega_{+} \leq \Omega \leq \Omega_{-}$[44], where

$$
\Omega_{ \pm}=\frac{-g_{t \phi} \pm \sqrt{g_{t \phi}^{2}-g_{t t} g_{\phi \phi}}}{g_{\phi \phi}}
$$

On the boundary of ergoshpere, $g_{t t}=0$ and $\Omega_{+}=0$, while inside the ergoshpere, $g_{t t}<0$ and $\Omega_{ \pm}>0$, and every particle move in the direction of BH rotation [28, 45]. For the brane Kerr BH, on substituting the values of $g_{t t}, g_{t \phi}$ and $g_{\phi \phi}$ form Eq. (2) into Eq. (12), we have

$$
\Omega_{ \pm}=\frac{a \sin \theta(b-2 M r) \pm \Sigma \sqrt{\Delta}}{\sin \theta\left[a^{2} \sin ^{2} \theta \Delta-\left(r^{2}+a^{2}\right)^{2}\right]}=\omega \pm \frac{\Sigma \sqrt{\Delta}}{\sin \theta\left[a^{2} \sin ^{2} \theta \Delta-\left(r^{2}+a^{2}\right)^{2}\right]},
$$

where

Approaching the event horizon, we obtain

$$
\omega=\frac{a(b-2 M r)}{a^{2} \sin ^{2} \theta \Delta-\left(r^{2}+a^{2}\right)^{2}} .
$$

$$
\lim _{r \rightarrow r_{+}} \Omega_{+}=\lim _{r \rightarrow r_{+}} \Omega_{-}=\omega_{b h}=\frac{a\left(2 M r_{+}-b\right)}{r_{+}^{4}+a^{2} r_{+}^{2}-a^{2}\left(2 M r_{+}-b\right)} .
$$

Here, $\omega_{b h}$ denotes angular velocity of the BH rotation and the angular velocity of particle turns out to be

$$
\Omega=\frac{d \phi}{d t}=\frac{\left(r^{2}-2 M r+b\right) L+a E(2 M r-b)}{\left[r^{2}\left(r^{2}+a^{2}\right)+a^{2}(2 M r-b)\right] E-a L(2 M r-b)} .
$$

The specific energy, as well as the specific angular momentum of circular orbits at a given radius $r$, can be written as [46]

$$
\begin{aligned}
E & =\frac{\left(r^{2}+b-2 M r \pm a \chi\right)}{r\left[r^{2}+2 b-3 M r \pm 2 a \chi\right]^{1 / 2}}, \\
L & = \pm \frac{\left(\left(r^{2}+a^{2} \mp 2 a \chi\right) \mp b a\right)}{r\left[r^{2}+2 b-3 M r \pm 2 a \chi\right]^{1 / 2}},
\end{aligned}
$$

where $\chi=\sqrt{M r-b}$, while the upper and lower signs correspond to the corotating and counter-rotating orbits, respectively. Substituting Eqs. (15) and (16) into (14), we obtain

$$
\Omega=\frac{\mp \sqrt{M r-b}}{\left(r^{2} \mp a \sqrt{M r-b}\right)} .
$$

The graphical behavior of angular velocity $\Omega$ is depicted in Fig. 3 It is observed that angular velocity decreases as the brane parameter $b$ increases (both upper and lower row left panel), whereas increases as the brane parameter $b$ increases (both upper and lower row right panel). 

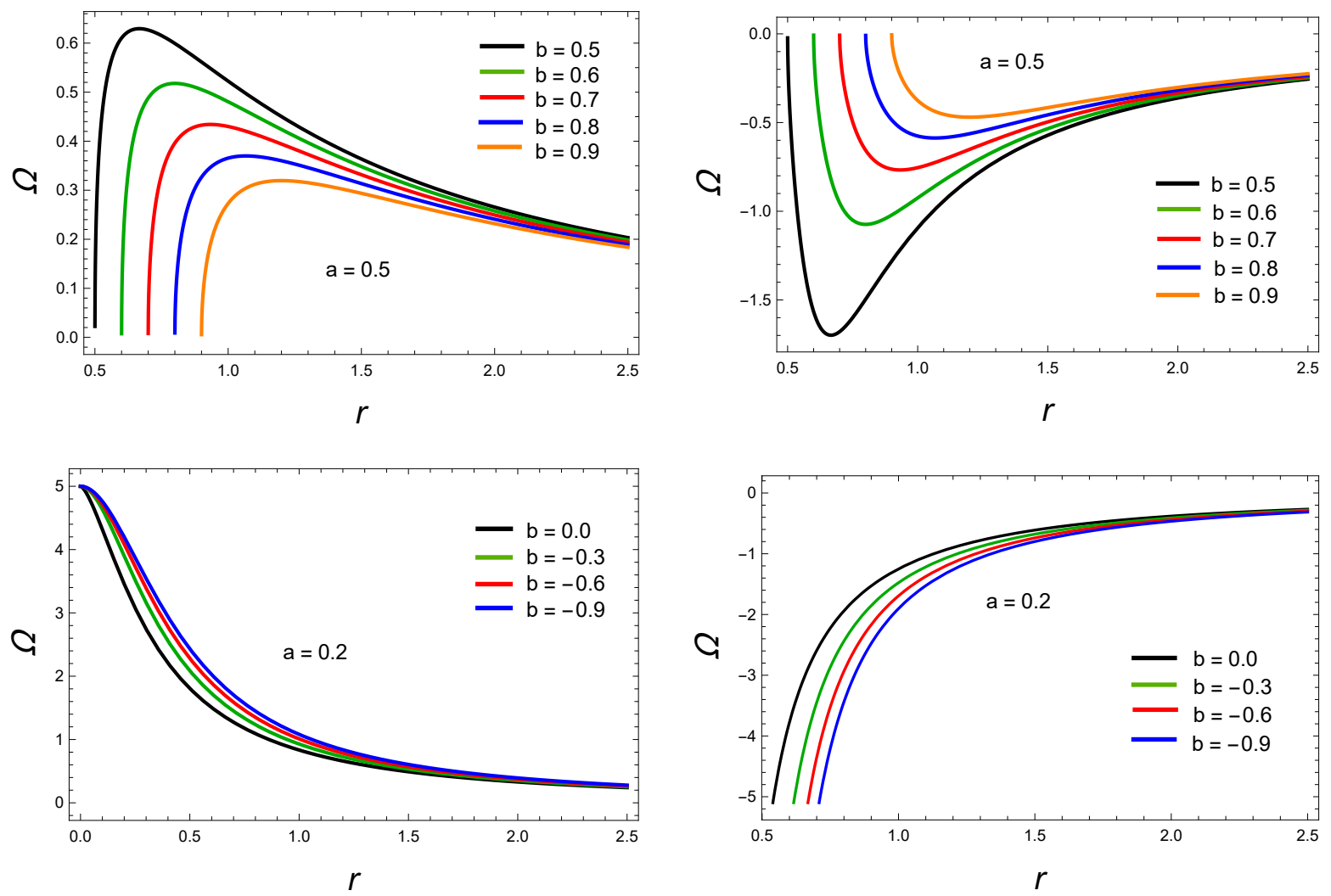

Figure 3: The behavior of angular velocity $\Omega$ as a function of $b$ verses $r$.

\section{Collisional Process in Ergoregion}

The collisional process in rotating and charged BHs is an important and interesting issue of GR. During the collision process, energy can be extracted from a $\mathrm{BH}$. There are a number of different techniques that can be used to explore the process of energy extraction from a rotating BH. Penrose process [33] is among the most rigorous and efficient energy extraction techniques than those of the nuclear reactions. In this mechanism, a particle enters into ergosphere having positive energy, subdivided into two particles, one of them follows the trajectory of negative energy whereas the other escape to infinity having more energy as compared to the incident ones. If the particles are involved in the Penrose process then the necessary and sufficient condition to extract energy from a BH is the absorption of particles with negative energies as well as angular momentum.

\subsection{Negative Energy States}

The negative energy states may occur due to the counter-rotating orbits (similar to that of the Kerr BH) as well as due to the electromagnetic interactions (similar to that of the $\mathrm{RN} \mathrm{BH}$ ) [42]. It is of great interest to find out the energy limits of a particle which they have at a particular location. The radial equation (10) could be rewritten as

$$
\begin{aligned}
E^{2}\left[\left(r^{2}\right.\right. & \left.\left.+a^{2}\right) r^{2}+a^{2}(2 M r-b)\right]-2 a E L(2 M r-b) \\
& -L^{2}\left(r^{2}-2 M r+b\right)+r^{2} \varepsilon \Delta=0 .
\end{aligned}
$$

The values of $E$ and $L$ can be obtained from the above equation as

$$
\begin{aligned}
E & =\frac{a L(2 M r-b) \pm \mathscr{Z}_{1} \sqrt{\Delta}}{r^{4}+a^{2}\left(r^{2}+2 M r-b\right)}, \\
L & =\frac{-a E(2 M r-b) \pm \mathscr{Z}_{2} \sqrt{\Delta}}{\left(r^{2}-2 M r+b\right)},
\end{aligned}
$$



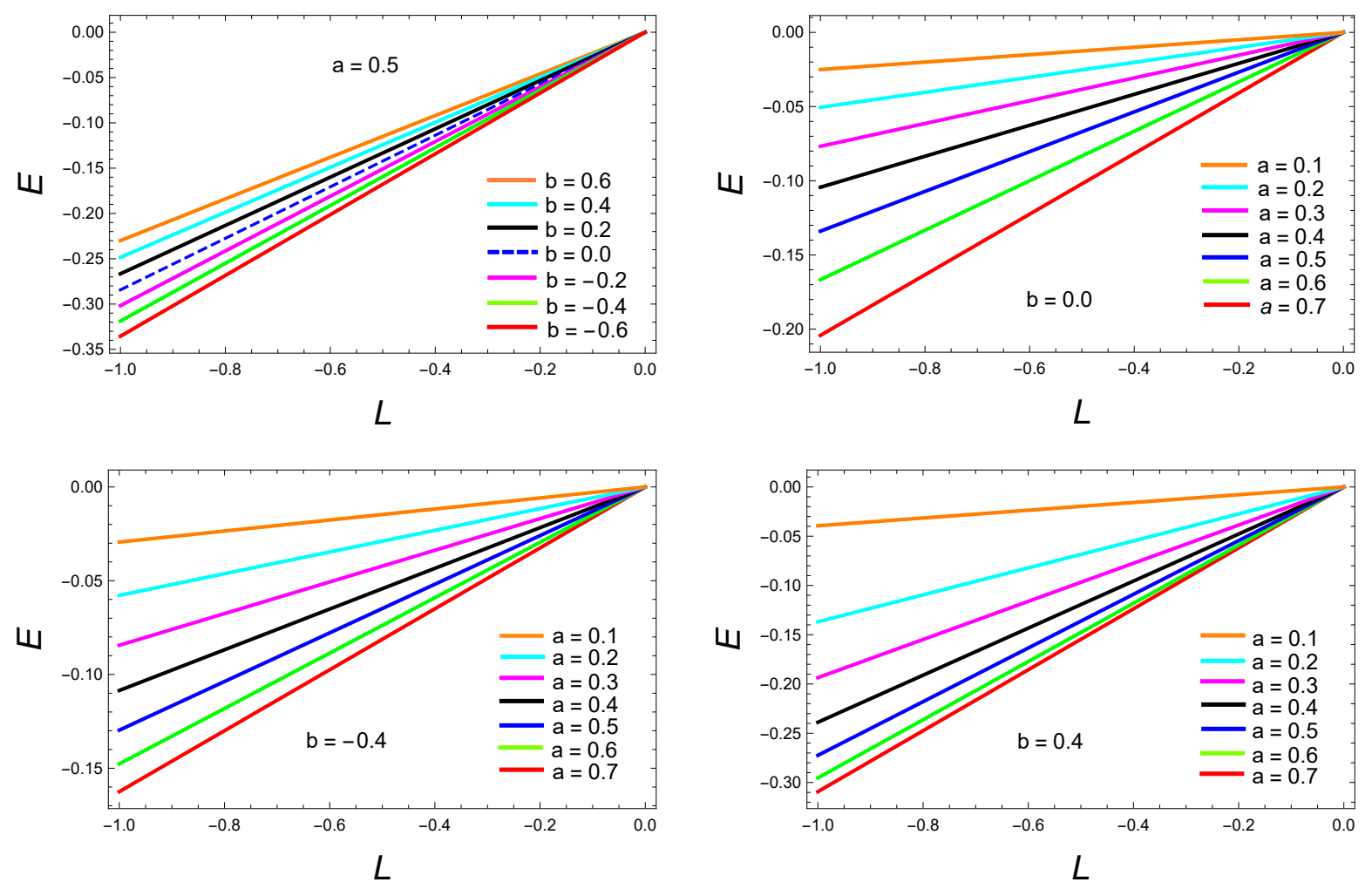

Figure 4: Graphical representation of the negative energy states $E$ versus the angular momentum $L$.

where

$$
\begin{aligned}
& \mathscr{Z}_{1}=\sqrt{L^{2} r^{4}-\left[r^{4}+a^{2}\left(r^{2}+2 M r-b\right)\right] \varepsilon r^{2}}, \\
& \mathscr{Z}_{2}=\sqrt{r^{4} E^{2}+\left(r^{2}-2 M r+b\right) \varepsilon r^{2}} .
\end{aligned}
$$

The following expression has been used to obtain the above results

$$
r^{4} \Delta-a^{2}(2 M r-b)^{2}=\left(r^{4}+a^{2}\left(r^{2}+2 M r-b\right)\right)\left(r^{2}-2 M r+b\right) .
$$

The conditions for negative energy can be deduced from Eq. [19). We set an energy $E=1$ and choose + sign of Eq. 19.). For $E<0$, it also necessary that $L<0$, and

$$
a^{2} L^{2}(2 M r-b)^{2}>r^{2} \Delta\left[r^{2} L^{2}-\left(r^{4}+a^{2}\left(r^{2}+2 M r-b\right)\right) \varepsilon\right] .
$$

Using Eq. 21], expression (22) can be written as

$$
\left[r^{4}+a^{2}\left(r^{2}+2 M r-b\right)\right]\left[\left(r^{2}-2 M r+b\right) L^{2}-\varepsilon r^{2} \Delta\right]<0 .
$$

It can be concluded from inequality 23 that $E<0 \Leftrightarrow L<0$, and

$$
\left(\frac{r^{2}-2 M r+b}{r^{2}}\right)<\frac{\Delta \varepsilon}{L^{2}}
$$

For $b=0$, the above inequality reduced to the case of Kerr BH [45]. The behavior of negative energy $E$ versus angular momentum $L$ is illustrated in Fig. 4 The top left graph shows an increase in negative energy $E$ for decreasing values of $b$. The behavior of negative energy at $b=0, b=-0.4$ and $b=0.4$ are described in the top right, bottom left and bottom right panels respectively, at different values of the spin parameter $a$. Furthermore, it is concluded that the BH negative energy increases with the increase of its rotation and brane parameter $b$.

The irreducible mass of a BH is one of the consequences of energy extraction from the BH. When a particle of negative energy enters into the $\mathrm{BH}$, the mass of the $\mathrm{BH}$ changes by a quantity $\delta M=E$ [47]. There is no upper bound on $\delta M$ 
as it can be increased by increasing mass of the injected particle. However, there is a lower bound on $\delta M$ and each incident particle having negative energy decreases the mass of the $\mathrm{BH}$ until its irreducible mass. The lower bound of $\delta M$ can be found using Eq. 19. At horizon, the discriminant of Eq. 19] is zero and thus we obtained the lower limit

$$
\delta M=\frac{a L\left(2 M r_{+}-b\right)}{r_{+}^{4}+a^{2}\left(r_{+}^{2}+2 M r_{+}-b\right)} .
$$

From Eq. 25, it is concluded that to extract energy from a $\mathrm{BH}$, the injected particle must have negative angular momentum and the brane parameter $b$ effects the value of $\delta M$.

\subsection{The Wald Inequality}

It is very important to study the limits of energy extraction by the Penrose process. Wald [48] derived an inequality which can discuss the limitations of energy extraction by this process. To obtain these limits for the brane Kerr BH, consider a particle with specific energy $\mathscr{E}$ and four velocity $U^{\alpha}$, breaks up into fragments. Let $\varepsilon$ be the specific energy and $u^{\alpha}$ be the four-velocity of one of the fragments. To derive the limits of $\varepsilon$, consider an orthonormal tetrad frame $e_{j}^{\alpha}$, in which $U^{\alpha}$ coincides with $e_{0}^{\alpha}$ and the remaining basis vectors are $e_{(i)}^{\alpha}(i=1,2,3)$. In this frame

$$
u^{\alpha}=\rho\left(U^{\alpha}+v^{(i)} e_{(i)}^{\alpha}\right)
$$

where $v^{(i)}$ are the spatial components of the three velocity of the fragment $\rho=1 / \sqrt{1-|v|^{2}}$ and $|v|^{2}=v^{(i)} v_{(i)}$. Since spacetime allows the time like Killing vector $\xi_{\alpha}=\partial / \partial x^{0}$, it can be represented in the tetrad frame as

$$
\xi_{\alpha}=\xi_{(0)} U_{\alpha}+\xi_{(i)} e_{\alpha}^{(i)}
$$

Now, the energy $\mathscr{E}$ in terms of the Killing vector can be written as

$$
\mathscr{E}=\xi_{\alpha} U^{\alpha}=\xi_{(0)}=\xi^{\alpha} U_{\alpha}=\xi^{(0)},
$$

and

Thus, we have the relation

$$
g_{00}=\xi^{\alpha} \xi_{\alpha}=-\xi_{(0)}^{2}+\xi_{(i)} \xi^{(i)}=-\mathscr{E}^{2}+|\xi|^{2}
$$

$$
|\xi|^{2}=\xi_{(i)} \xi^{(i)}=\mathscr{E}^{2}+g_{00}
$$

From Eq. 26, we obtain

$$
\varepsilon=\xi_{(\alpha)} u^{(\alpha)}=\rho\left(\xi_{(0)}+v^{(i)} \xi_{(i)}\right)=\rho(\mathscr{E}+|v||\xi| \cos \theta),
$$

where $\theta$ is the angle between $v^{(i)}$ and $\xi_{(i)}$. Using Eq. 30, one can rewrite Eq. 31 in the following form

$$
\varepsilon=\rho \mathscr{E}+\rho|v| \sqrt{\mathscr{E}^{2}+g_{00}} \cos \theta
$$

Equation 32 provides the inequality

$$
\rho \mathscr{E}-\rho|v| \sqrt{\mathscr{E}^{2}+g_{00}} \leq \varepsilon \leq \rho \mathscr{E}+\rho|v| \sqrt{\mathscr{E}^{2}+g_{00}}
$$

For the brane Kerr BH, the Wald inequality can be written in the following form

$$
\rho \mathscr{E}-\rho|v| \sqrt{\mathscr{E}^{2}+1-b} \leq \varepsilon \leq \rho \mathscr{E}+\rho|v| \sqrt{\mathscr{E}^{2}+1-b} .
$$

For $a=\sqrt{2} M$ and $b=-M^{2}$, radius of the stable innermost circular orbit is located at $M$, i.e., $r_{I S C O} \simeq M$ [43]. Substituting the value of $r_{I S C O}$ in Eq. 12, we can obtain the maximum energy for a particle orbiting a stable circular orbit

$$
\mathscr{E}_{0}=\frac{1}{\sqrt{3-b}} .
$$

For $\varepsilon$ to be negative, it is necessary that

$$
|v|>\frac{\mathscr{E}}{\sqrt{\mathscr{E}^{2}+1-b}}=\frac{1}{2-b} .
$$

Otherwise, the fragments should have relativistic energies which could be possible before the energy extraction. For $b=0$, Eq. (36) reduces to the Kerr BH [45]. 


\subsection{Efficiency of The Process}

The efficiency of energy extraction from a $\mathrm{BH}$ via the Penrose process is among the principal consequences in the energetics of BHs. Consider a particle bearing energy $E^{(0)}$ enters into the ergosphere of a BH and subdivide into two particles namely 1 and 2, (bearing energy $E^{(1)}$ and $E^{(2)}$, respectively). The particle 1 has more energy as compared to the incident ones and exits the ergosphere whereas, particle 2 bearing negative energy falls into the $\mathrm{BH}$. Using the law of energy conservation

$$
E^{(0)}=E^{(1)}+E^{(2)},
$$

here $E^{(2)}<0$, implies that $E^{(1)}>E^{(0)}$. Let $v=d r / d t$ be the radial velocity of a particle with respect to an observer at infinity. Using the laws of conservation of angular momentum and energy

$$
L=p^{t} \Omega, \quad E=-p^{t} Y,
$$

where

$$
Y \equiv g_{t t}+g_{t \phi} \Omega
$$

Using $p^{\eta} p_{\eta}=-m^{2}$, we have

$$
g_{t t} \dot{t}^{2}+2 g_{t \phi} \dot{t} \dot{\phi}+g_{r r} \dot{r}^{2}+g_{\phi \phi} \dot{\phi}^{2}=-m^{2}
$$

Dividing Eq. 39 by $\dot{t}^{2}$, we obtain

$$
g_{t t}+2 \Omega g_{t \phi}+\Omega^{2} g_{\phi \phi}+\frac{v^{2}}{\Delta} r^{2}=-\left(\frac{m Y}{E}\right)^{2} .
$$

Since the fourth term, in left hand side of Eq. (40) is always positive and the right hand side is negative or equal to zero. Hence, we can rewrite Eq. (40) in the following form

$$
g_{t t}+2 \Omega g_{t \phi}+\Omega^{2} g_{\phi \phi}=-\left(\frac{m Y}{E}\right)^{2}-\frac{v^{2}}{\Delta} r^{2} \leq 0 .
$$

Using Eq. [37), the relation of conservation of angular momentum and energy could be expressed as

$$
\begin{aligned}
& p_{(0)}^{t} Y_{(0)}=p_{(1)}^{t} Y_{(1)}+p_{(2)}^{t} Y_{(2)}, \\
& p_{(0)}^{t} \Omega_{(0)}=p_{(1)}^{t} \Omega_{(1)}+p_{(2)}^{t} \Omega_{(2)} .
\end{aligned}
$$

Henceforth, efficiency $(\eta)$ of the collisional Penrose process can be described as

$$
\eta=\frac{E^{(1)}-E^{(0)}}{E^{(0)}}=\chi-1,
$$

in which $\chi=E^{(1)} / E^{(0)}$ and $\chi>1$. By the use of Eqs. 37, , 42, and 443 , we acquire

$$
\chi=\frac{E^{(1)}}{E^{(0)}}=\frac{\left(\Omega_{(0)}-\Omega_{(2)}\right) Y_{(1)}}{\left(\Omega_{(1)}-\Omega_{(2)}\right) Y_{(0)}} .
$$

Let us consider an incident particle having energy $E^{(0)}=1$ enters into the ergosphere and subdivided into two photons having momenta $p^{(1)}=p^{(2)}=0$. It can be observed from Eq. 45, that efficiency could be maximized by having the smallest value of $\Omega_{(1)}$ and the largest value of $\Omega_{(2)}$ concurrently, which required $v_{(1)}=v_{(2)}=0$. For this case

$$
\Omega_{(1)}=\Omega_{+}, \quad \Omega_{(2)}=\Omega_{-} .
$$

The corresponding values of parameter $Y$ are

$$
Y_{(0)}=g_{t t}+\Omega_{(0)} g_{t \phi}, \quad Y_{(2)}=g_{t t}+\Omega_{-} g_{t \phi} .
$$

The four-momenta of pieces are

$$
p_{\eta}=p^{t}\left(1,0,0, \Omega_{\eta}\right), \quad \eta=1,2 .
$$

Accordingly, Eq. 39] could be written as

$$
\left(g_{t \phi}^{2}+g_{\phi \phi}\right) \Omega^{2}+2 \Omega\left(1+g_{t t}\right) g_{t \phi}+\left(1+g_{t t}\right) g_{t t}=0 .
$$


SAEED Ullah Khan, Jingli ReN

Table 2: Maximum efficiency $\eta_{\max }(\%)$ of the energy extraction from BH via the Penrose process.

\begin{tabular}{cccccccc}
\hline \hline & $\mathrm{a}=0.2$ & $\mathrm{a}=0.4$ & $\mathrm{a}=0.6$ & $\mathrm{a}=0.8$ & $\mathrm{a}=0.9$ & $\mathrm{a}=0.99$ & $\mathrm{a}=1.0$ \\
\hline$b=-0.5$ & 0.2046 & 0.8520 & 2.0625 & 4.1361 & 5.7156 & 7.6822 & 7.9470 \\
$b=-0.2$ & 0.2313 & 0.9711 & 2.3930 & 4.9859 & 7.1611 & 10.2955 & 10.7754 \\
$b=-0.1$ & 0.2422 & 1.0208 & 2.5365 & 5.3903 & 7.9267 & 12.0638 & 12.7936 \\
$b=0.0$ & 0.2545 & 1.0774 & 2.7046 & 5.9017 & 9.0098 & 16.1956 & 20.7107 \\
$b=0.005$ & 0.2551 & 1.0805 & 2.7138 & 5.9310 & 9.7713 & 16.6794 & \\
$b=0.01$ & 0.2558 & 1.0835 & 2.7231 & 5.9608 & 9.1461 & 17.2818 & \\
$b=0.02$ & 0.2571 & 1.0897 & 2.7419 & 6.0216 & 9.2894 & & \\
$b=0.1$ & 0.2685 & 1.1429 & 2.9059 & 6.5846 & 10.8130 & & \\
$b=0.2$ & 0.2846 & 1.2197 & 3.1536 & 7.5876 & & & \\
$b=0.5$ & 0.3538 & 1.5714 & 4.5583 & & & & \\
\hline \hline
\end{tabular}

Consequently, from the aforementioned equation, the angular velocity of the incident particle will take the form

$$
\Omega_{(0)}=\frac{-\left(1+g_{t t}\right) g_{t \phi}+\sqrt{\left(1+g_{t t}\right)\left(g_{t \phi}^{2}-g_{\phi \phi} g_{t t}\right)}}{g_{t \phi}^{2}+g_{\phi \phi}} .
$$

Substituting Eqs. (46) and (47) into 45), the efficiency of the energy extraction can be obtained as

$$
\eta=\frac{\left(g_{t t}+g_{t \phi} \Omega_{+}\right)\left(\Omega_{(0)}-\Omega_{-}\right)}{\left(g_{t t}+g_{t \phi} \Omega_{0}\right)\left(\Omega_{(+)}-\Omega_{-}\right)}-1
$$

In order to acquire maximum efficiency $\left(\eta_{\max }\right)$, it is necessary for the incident particle to be subdivided near the horizon of a BH. Henceforth, the aforementioned equation turns out to be

$$
\eta_{\max }=\frac{1}{2}\left(\sqrt{\frac{\left(2 M r_{+}-b\right)}{r_{+}^{2}}}-1\right) .
$$

The numerically calculated values for maximum efficiency of the energy extraction via the Penrose process at different choices of the brane parameter $b$ and spin $a$, are shown in Table 2. It is described that maximum efficiency of the mechanism could be achieved by increasing the values of the brane parameter $b$. For $a=1$ and $b=0$, the maximum efficiency could be $20.7 \%$, i.e., the limiting value for extreme Kerr BH [45]. Rotation of the BH has a great influence on the motion and particle collision. The rapid rotation of a $\mathrm{BH}$ contributes gain in its energy extraction. The behavior of the maximum efficiency of energy extraction via the Penrose process is also depicted in Fig. 5 at different values of rotation as well as the tidal charge parameter $b$. The dashed curve corresponds to the Kerr BH. It could be observed that for both negative as well as positive values of $b$, the efficiency of energy extraction increases with brane parameter $b$ of the $\mathrm{BH}$. It is noted that rotation of the $\mathrm{BH}$ tends to increase the efficiency of energy extraction.

\section{Concluding Remarks}

In this article, particle motion and the collisional Penrose process in ergoregion of the braneworld Kerr BH has been explored. The circular motion of the test particle plays an essential role to understand the accretion disk theory. Geodesics are important to study the particle motion and dynamics of galaxies. We have explored the geodesics for particles orbiting the braneworld Kerr BH. The properties of event horizon, static limit and ergoregion of the brane Kerr $\mathrm{BH}$ are explored in detail. For both positive as well as negative tidal charge, the possible situations are discussed. It is worthily to mention that the negative tidal charge could provide a mechanism to spin up the $\mathrm{BH}$ for which the rotation parameter must be greater than the mass of the BH but in the scenario of GR, this situation is not allowed. It is observed that both the spin as well as the tidal charge, effects the area of ergoregion. The sign of tidal charge also influences the shape of ergoregion and event horizon. For $b>0$, the ergoregion becomes thick, consequently, the static limit and horizon decreases. For $b<0$, the boundary of ergosphere extends which makes the $\mathrm{BH}$ more energetic with respect to the extraction of energy.

Energy extraction from the braneworld Kerr $\mathrm{BH}$ via the Penrose process has been studied. We have also examined the negative energy states, irreducible mass of the $\mathrm{BH}$ and limitations of the energy extraction by the Wald inequality. It is observed that negative energy is allowed only for negative angular momentum. It is found that both the negative tidal 

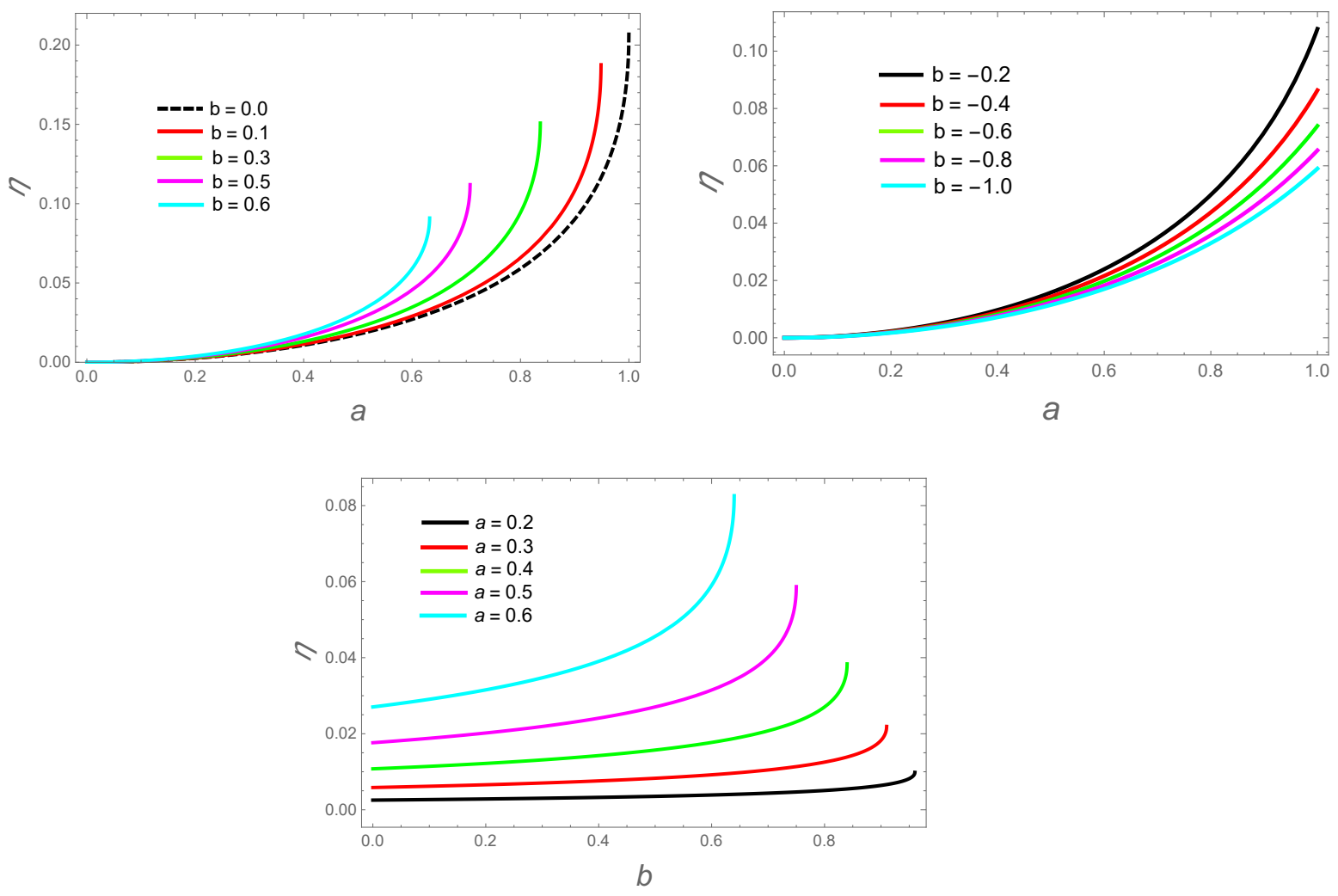

Figure 5: Maximum efficiency of the energy extraction as a function of $a$ (top) whereas $b$ (bottom).

charge and rotation of a $\mathrm{BH}$ tends to increase its negative energy while in case of the positive tidal charge, the particles have more negative energy. In case of the Kerr BH, particles have more negative energy as compared to the brane Kerr BH with negative tidal charge.

We have explored the efficiency of energy extraction from the brane Kerr BH. It is concluded that for both positive as well as negative values of the tidal charge, efficiency of the energy extraction increases. Rotation of the $\mathrm{BH}$ has great influence on particles motion and collision. In case of rapid rotation, particles can take energy from BH rotation as a result, more energy can be extracted. Our obtained results are much similar to that of the Kerr BH surrounded by magnetic field as in both situations, the efficiency is greatly influenced by the BH rotation [41]. Moreover, in the case of magnetic Penrose process, the magnetic effects increase the efficiency just like in brane Kerr BH does by the brane parameter $b$. In comparison with Kerr $\mathrm{BH}$, more energy can be extracted in case of brane Kerr $\mathrm{BH}$, but the maximum efficiency limit can be obtained for the extreme Kerr BH, i.e., 20.7\%.

\section{Acknowledgment}

J. L. Ren is very grateful for the financial support from the National Nature Science Foundation of China (11771407).

\section{References}

[1] Hořava, P., and Witten, E.: Nucl. Phys. B 475 (1996) 94.

[2] Hořava, P., and Witten, E.: Nucl. Phys. B 460 (1996) 506.

[3] Arkani-Hamed, N., Dimopoulos, S., and Dvali, G.: Phys. Lett. B 429 (1998) 263.

[4] Randall, L., and Sundrum, R.: Phys. Rev. Lett. 83 (1999) 3370.

[5] Dimopoulos, S., and Landsberg, G.: Phys. Rev. Lett. 87 (2001) 161602.

[6] Emparan, R., Masip, M., and Rattazzi, R.: Phys. Rev. D 65 (2002) 064023.

[7] Gimon, E. G., and Hořava, P.: Phys. Lett. B 672 (2009) 299. 
[8] Kološ, M., Stuchlík, Z., and Tursunov, A.: Class. Quantum Gravt. 32 (2015) 165009;

[9] Kološ, M., Tursunov, A., and Stuchlík, Z.: Eur. Phys. J. C 77 (2017) 860.

[10] Sharif, M. and Shahzadi, M.: Eur. Phys. J. C 77 (2017) 363.

[11] Sharif, M. and Shahzadi, M.: J. Exp. Theor. Phys. 127 (2018) 491.

[12] Oteeva, T., Kološ, M. and Stuchlík, Z.: Eur. Phys. J. C 78 (2018) 261.

[13] Dadhich, N., Maartens, R., Papadopoulos, P., and Rezania, V.: Phys Lett. B 487 (2000) 1.

[14] Kotrlová, A., Stuchlík, Z., and Török, G.: Class. Quantum Gravt. 25 (2008) 225016.

[15] Pugliese, D., Quevedo, H., and Ruffini, R.: Phys. Rev. D 83 (2011) 024021.

[16] Pugliese, D., Quevedo, H., and Ruffini, R.: Phys. Rev. D 83 (2011) 104052.

[17] Pugliese, D., Quevedo, H., and Ruffini, R.: Phys. Rev. D 88 (2013) 024042.

[18] Stuchlík, Z., and Kološ, M.: J. Cosmol. Astropart. Phys. 2012 (2012) 008.

[19] Casadio, R., and Ovalle, J.: Gen. Relativ. Gravit. 46 (2014) 1669.

[20] Blaschke, M., and Stuchlík, Z.: (2016). Phys. Rev. D 94 (2016) 086006.

[21] Stuchlík, Z., Blaschke, M., and Schee, J.: Phys. Rev. D 96 (2017) 104050.

[22] Stuchlík, Z., Hledík, S.: Acta Physica Slovaca 52 (2002) 363.

[23] Grib, A. A. E., and Pavlov, Y. V.: Theor. Math. Phys. 190 (2017) 268.

[24] Nakao, K. I., et al.: Phys. Lett. B 780 (2018) 410.

[25] Toshmatov, B., Stuchlík, Z., Schee, J., and Ahmedov, B.: Phys. Rev. D 93 (2016) 124017.

[26] Stuchlík, Z., and Kološ, M.: Eur. Phys. J. C 76 (2016) 32.

[27] Zeldovich, I. B., et al.: Relativistic astrophysics, 2: The structure and evolution of the Universe (Vol. 2)., University of Chicago Press (1971).

[28] Misner, C. W., Thorne, K. S., and Wheeler, J. A.: Gravitation (1973).

[29] Keeton, C. R., and Petters, A. O.: Phys. Rev. D 73 (2006) 104032.

[30] Böhmer, C. G., Harko, T., and Lobo, F. S.: Class. Quantum Gravt. 25 (2008) 045015.

[31] Schee, J., and Stuchlík, Z.: Int. J. Mod. Phys. D 18 (2009) 983.

[32] Schee, J., and Stuchlík, Z.: Gen. Relativ. Gravit. 41 (2009) 1795.

[33] Penrose, R.: Nuovo Cimento Riv. Ser. 1 (1969) 252;

Penrose, R., and Floyd, R. M.: Nat. Phys. Sci. 229 (1971) 177.

[34] Nozawa, M., and Maeda, K. I.: Phys. Rev. D 71 (2005) 084028.

[35] Mukherjee, S.: Phys. Lett. B 778 (2018) 54.

[36] Bhat, M., Dhurandhar, S., and Dadhich, N.: J. Astrophys. Astron. 6 (1985) 85.

[37] Parthasarathy, S., Wagh, S. M., Dhurandhar, S. V., and Dadhich, N.: Astrophys. J. 307 (1986) 38.

[38] Liu, C., Chen, S., and Jing, J.: Astrophys. J. 751 (2012) 148.

[39] Toshmatov, B., et al.: Astrophys. Space Sci. 357 (2015) 41.

[40] Liu, Y., and Liu, W. B.: Phys. Rev. D 97 (2018) 064024.

[41] Dadhich, N., Tursunov, A., Ahmedov, B., and Stuchlík, Z.: Mon. Notices Royal Astron. Soc. Lett. 478 (2018) L89.

[42] Shahzadi, M., Yousaf, Z., and Khan, S. U.: Phys. Dark Universe 24 (2019) 100263, arXiv:1812.11036 [gr-qc]

[43] Aliev, A. N., and Gümrükçüoğlu, A. E.: Phys. Rev. D 71 (2005) 104027.

[44] Lightman, A. P., et al.: Problem book in relativity and gravitation., Princeton University Press (2017).

[45] Chandrasekhar, S.: The Mathematical Theory of Black Holes, Oxford University Press (1983).

[46] Stuchlík, Z., and Kotrlová, A.: Gen. Relativ. Gravit. 41 (2009) 1305.

[47] Abdujabbarov, A. A., et al.: Astrophys. Space Sci. 334 (2011) 237.

[48] Wald, R. M.: Astrophys. J. 191 (1974) 231. 